\title{
Correlation-induced spectral changes in tissues
}

\author{
Ruoyu Zhu, ${ }^{1}$ Shamira Sridharan, ${ }^{1}$ Krishnarao Tangella, ${ }^{2}$ Andre Balla, ${ }^{3}$ and Gabriel Popescu ${ }^{1, *}$ \\ ${ }^{1}$ Quantitative Light Imaging Laboratory, Department of Electrical and Computer Engineering, Beckman Institute for \\ Advanced Science \& Technology, University of Illinois at Urbana-Champaign, Urbana, Illinois 61801 USA \\ ${ }^{2}$ Department of Pathology, Christie Clinic and University of Illinois at Urbana-Champaign, Urbana, Illinois 61801, USA \\ ${ }^{3}$ Department of Pathology, University of Illinois at Chicago, Chicago, Illinois 60612 USA \\ ${ }^{*}$ Corresponding author: gpopescu@illinois.edu
}

Received July 26, 2011; revised August 31, 2011; accepted September 9, 2011;

posted September 28, 2011 (Doc. ID 151772); published October 25, 2011

\begin{abstract}
We report experimental evidence of correlation-induced spectral changes in biological tissues. The overall spectral shift in our transmission measurements is to the red and the mean wavelength of the original spectrum is up $10 \%$ larger. These results indicate that the spectral changes due to elastic scattering are significant and likely to hinder all spectroscopic measurements based on the inelastic (i.e., emission and absorption) interaction between light and tissues. Thus, simultaneous morphology and spectral measurements are required for accurate measurements spectroscopic information. (c) 2011 Optical Society of America

OCIS codes: $\quad 030.1670,030.1640,170.3660,170.6510$.
\end{abstract}

In 1986, Wolf predicted theoretically that spatial correlations in a primary source can affect the optical spectrum in the far-zone, unless the complex degree of coherence obeys a certain scaling law [1]. These predictions were first confirmed experimentally using acoustic waves [2]. Later, it was shown that the correlation-induced spectral shifts, sometimes called Wolf shifts, may affect spectroscopic measurements on stellar objects such as quasars [3]. Similar effects have been reported later in the case of spatially correlated secondary sources, i.e., scattering media $[\underline{4}, 5]$. Wolf, Foley, and Gori emphasized the surprising nature of these results [4]: "It is commonly stated that $[\ldots]$ the frequency of the incident light will not change by the process of scattering. How is it possible then that our analysis indicates that such scattering may produce frequency shifts of the spectral lines?" The explanation is that, although the medium does not absorb differently various spectral components of the incident broadband light, it scatters with different strengths the various wavelengths, depending on the scattering angle. Thus, the scattering process essentially redistributes the spectral energy at each scattering angle.

Previous work by Gao has shown theoretically that light scattering through tissue samples induces spectral changes [6,7]. It that case, a generic refractive index spatial correlation function was postulated, rather than measured. Hunter et al. showed that tissue spatial correlation function can be used for diagnosis of cancer [8].

In this Letter, we present experimental evidence of correlation-induced spectral changes in biological tissues by measuring the spatial correlation function associated with an actual tissue slice. We show that the spectral changes due to elastic scattering are significant and likely to hinder all spectroscopic measurements based on the inelastic (i.e., emission and absorption) interaction between light and tissues. This effect should be especially important whenever quantitative spectroscopy is performed for tissue investigation and diagnosis [9]. One particular application of vibrational spectroscopy in tissues is cancer diagnosis in human biopsies, where accurate measurement of spectra is of essence [10].

We quantified the spatial correlations of tissue biopsies by performing high-transverse resolution quantitative phase imaging (QPI) of unstained histology slides [Fig. 1(a)]. QPI has been shown to provide sensitive angular scattering data, an approach referred to as Fourier transform light scattering [11]. Here we used spatial light interference microscopy (SLIM), a recent QPI method that can extract pathlength maps from tissues with spatial sensitivity of $0.3 \mathrm{~nm}$ [1ㅡ] . SLIM provides high-contrast, quantitative information from unstained tissues slices with subcellular resolution $[\underline{13}, 14]$. Figure $\underline{1(a)}$ shows the quantitative phase image of a prostate needle biopsy.

Figure 1(b) illustrates the scattering geometry, where the tissues slice is illuminated by a plane wave, $A(\omega) \exp \left(i k_{0} z\right)$, of spectral amplitude $A$, and incident wave vector $\mathbf{k}_{\mathbf{i}}=k_{0} \hat{\mathbf{z}}$. In order to calculate the optical spectrum associated with a scattering angle $\theta$, we start with the (inhomogeneous) Helmholtz equation that describes the scattered field as a function of angular frequency, $\omega$, and spatial coordinate, $\mathbf{r}$,
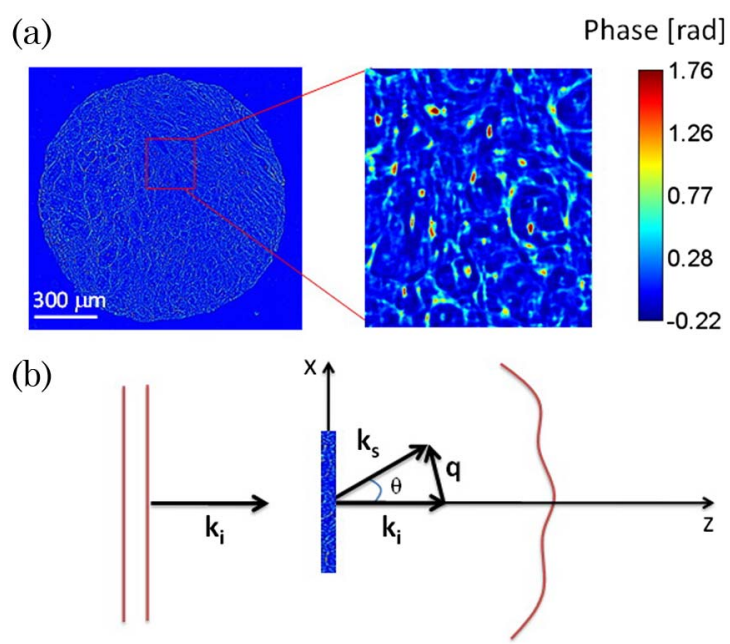

Fig. 1. (Color online) a) Quantitative phase image of a $4 \mu \mathrm{m}$ thick core biopsy. The inset shows the zoomed-in version of the boxed area. Color bar indicates phase shift in red. b) Experimental geometry for light scattering by thin tissue slice: $\mathbf{k}_{\mathbf{i}}, \mathbf{k}_{\mathbf{s}}, \mathbf{q}$, are the incident wavevector, scattered wavevector and momentum transfer. 


$$
\nabla^{2} U(\mathbf{r}, \omega)+k_{0}^{2} U(\mathbf{r}, \omega)=-k_{0}^{2} \chi(\mathbf{r}) U(\mathbf{r}, \omega) .
$$

In Eq. (1), $k_{0}=\omega / c, c$ is the speed of light in vacuum, $\chi(\mathbf{r})=\left[n^{2}(x, y)-1\right] \delta(z)$ is the dielectric susceptibility of an optically thin specimen, with $n$ the refractive index, assumed to be nondispersive. Next, we employ the Born approximation, which essentially states that the plane wave incident remains a plane wave inside the tissue, i.e., we replace the field $U$ on the right hand side by the incident field, $A(\omega) \exp \left(i k_{0} z\right)$,

$$
\nabla^{2} U(\mathbf{r}, \omega)+k_{0}^{2} U(\mathbf{r}, \omega)=-k_{0}^{2} \chi(x, y) \delta(z) A(\omega) e^{i k_{0} z} .
$$

In order to solve this Helmholtz equation we take its Fourier transform with respect to $\mathbf{r}$ and solve for the field in the wave vector representation, $\tilde{U}(\mathbf{k}, \omega)$,

$$
\tilde{U}(\mathbf{k}, \omega)=\frac{k_{0}^{2}}{k^{2}-k_{0}^{2}} A(\omega) \tilde{\chi}\left(\mathbf{k}-\mathbf{k}_{i}\right),
$$

where $k=|\mathbf{k}|$ is the modulus of the wave vector. Equation (3) is another expression for the basic result of diffraction tomography [15], which states that measuring the scattered field along the direction of the wave vector $\mathbf{k}$ provides information about the object's spatial frequency component $\mathbf{q}=\mathbf{k}-\mathbf{k}_{\mathbf{i}}$. The scattering angle is related to the modulus of this scattering wave vector, $\mathbf{q}$ (or momentum transfer), $q=2 k_{0} \sin (\theta / 2)$ [Fig. 1(b)]. Since $\tilde{\chi}\left(\mathbf{k}-\mathbf{k}_{\mathbf{i}}\right)$ only depends on $k_{x}$ and $k_{y}$, we introduce the notation. $k_{\perp}=\left(k_{x}^{2}+k_{y}^{2}\right)^{1 / 2}$.

By denoting $Q^{2}=\left(k_{0}^{2}-k_{\perp}^{2}\right)$, Eq. (3) becomes

$$
\tilde{U}(\mathbf{k}, \omega)=\frac{k_{0}^{2}}{2 Q}\left(\frac{1}{Q-k_{z}}+\frac{1}{Q+k_{z}}\right) A(\omega) \tilde{\chi}\left(k_{\perp}\right) .
$$

Thus, if we take the 1D inverse Fourier transform with respect to $k_{z}$, we obtain the following expression of the scattered field with spherical wave terms that are counter propagating,

$$
\widetilde{U}\left(\mathbf{k}_{\perp}, z, \omega\right)=\frac{k_{0}^{2}}{2} A(\omega) \tilde{\chi}\left(k_{\perp}\right)\left(\frac{e^{i Q \cdot z}}{Q}+\frac{e^{-i Q \cdot z}}{Q}\right) .
$$

By suppressing the term that corresponds to backscattering, i.e., $\exp (-i Q \cdot z) / Q$, we arrive at the expression for the complex analytic signal associated with the scattered field

$$
\tilde{U}\left(\mathbf{k}_{\perp}, z, \omega\right)=\frac{k_{0}^{2}}{2} A(\omega) \tilde{\chi}\left(k_{\perp}\right)\left(\frac{e^{i Q \cdot z}}{Q}\right) .
$$

Finally, we obtain the expression for the power spectrum, $S(\mathbf{k}, \omega)=|\tilde{U}(\mathbf{k}, \omega)|^{2}$, which can be further expressed in terms of scattering angle $\theta$ as

$$
S(\theta, \omega)=\left.\left(\frac{k_{0}}{2 \cos \theta}\right)^{2} \tilde{\chi}^{2}\left(k_{\perp}\right) S_{i}(\omega)\right|_{k_{0}=\omega / c},
$$

where $\cos (\theta)=Q / k_{0}, k_{\perp}=k_{0} \sin \theta$, and $S_{i}$ is the incident power spectrum. Equation (7) shows the anticipated result that the optical spectrum at a certain scattering angle, $\theta$, depends on both the incident spectrum and spatial power spectrum, $\tilde{\chi}^{2}\left(k_{\perp}\right)$, of the scattering tissue. Essentially, each spatial frequency of the medium can be interpreted as a sinusoidal grating that diffracts fields of longer wavelengths at larger angles; the Wolf effect is the average effect of this diffraction phenomenon.

We used Eq. (7) to obtain the spectrum of the light scattered by the tissue slice in Fig. 1(a), which is illuminated by a Gaussian spectrum centered at $4.0 \times$ $10^{15}(\mathrm{rad} / \mathrm{s})$ and of standard deviation $1 \times 10^{14}$. From the quantitative phase image and known thickness $(4 \mu \mathrm{m})$, we obtained the refractive index distribution, $n(x, y)$, the susceptibility function, $\chi(x, y)$, and spatial power spectrum, $\left|\tilde{\chi}\left(k_{x}, k_{y}\right)\right|^{2}$. The scattered spectrum at each angle $\theta$ is shown in Fig. 2(a). We observe clear modifications with respect to the original spectrum, with an overall trend of red spectral shifting. This result is consistent with previous reports on spectral shifts due to static scattering [5]. In order to quantify this overall shift, we evaluated the shift in the mean frequency $\left(\omega^{\prime}\right)$ with respect to original mean frequency $\left(\omega_{0}\right)$; for each scattering angle, $\beta(\theta)=\left(\omega_{0}^{\prime}-\omega_{0}\right) / \omega_{0}$. These results are summarized in Fig. 2. These red shifts are significant, attending a maximum of more than $10 \%$. To further prove that this shift is due entirely to the spatial correlations, we also plotted the spectrum in the absence of correlations, i.e. $\left|\tilde{\chi}\left(k_{x}, k_{y}\right)\right|^{2}=1$ and the resulting normalized spectra at different angles remain unmodified, as in the inset of in Fig. 2(b).

The most dominant spectroscopic characteristic of tissues in the visible range is due to the hemoglobin absorption. Therefore it is important to understand how the hemoglobin absorption spectrum is transformed by the scattering process. Figure 3 shows the modifications in the spectrum of both oxy- and deoxy-hemoglobin as a function of scattering angle. These spectral changes will occur if, for example, a blood vessel is observed through the thin slice of tissue, such as that shown in Fig. 1(a). Again, these Wolf effects on both oxy- and deoxy-hemoglobin spectra are significant, with a general tendency to shift to the red wavelengths.

Our results show that the tissue spatial correlations can significantly affect the measured spectrum of scattered light at various angles. These spatial correlations act as a spectral filter that modifies (or redistributes) the spectral features due to inelastic scattering, i.e.
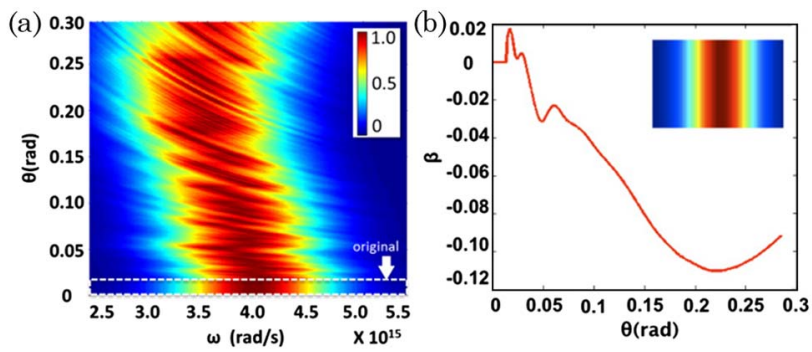

Fig. 2. (Color online) (a) Optical spectrum versus angle for light scattered by the tissue shown in Fig. 1(a). At small angles, the original Gaussian spectrum is shown. (b) The relative change of the mean frequency; negative values indicate red shifts. The inset shows the spectrum versus angle in the absence of spatial correlations. 

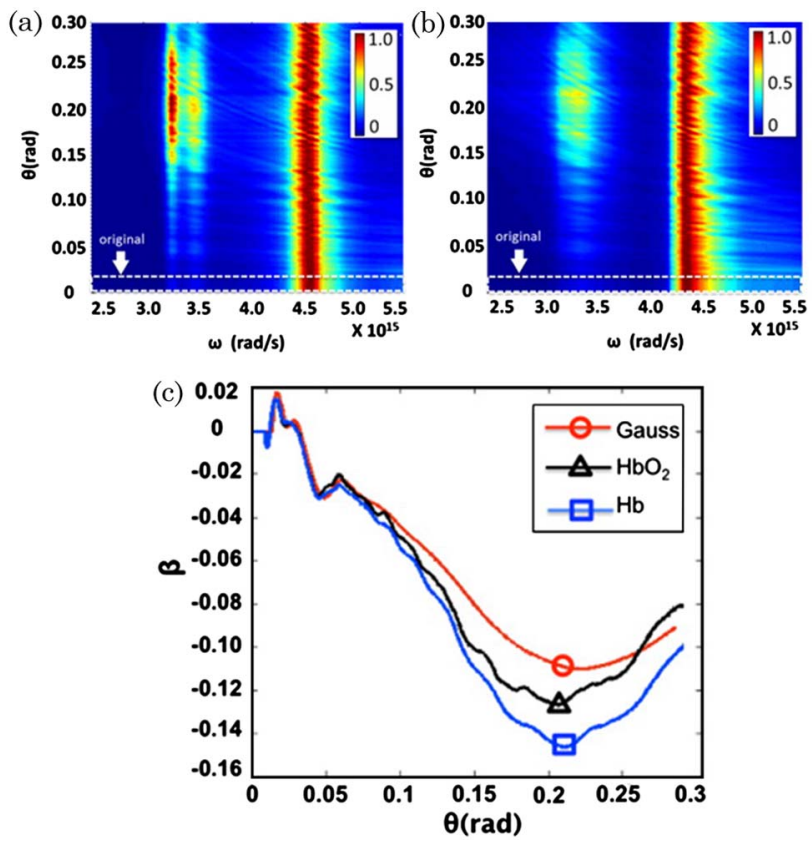

Fig. 3. (Color online) Optical spectrum versus scattering angle for (a) deoxy-hemoglobin and (b) oxy-hemoglobin. (c) The relative shift in mean frequency for the Gaussian, $\mathrm{Hb}$, and $\mathrm{HbO}_{2}$, as indicated.

spatial correlations would not affect the emission process, but would alter the shape of the emitted field. This effect is significant whenever angular and spectral resolved measurements are made. For example, the effect of Raman scattering can be easily outweighed by that of correlated scattering. As shown by our results, the overall shift is to the red and the mean wavelength of the original spectrum can be larger by approximately $10 \%$. However this is not a universal behavior. These spectral changes are due to redistributing the energy and not generating new light; therefore blue shifts can become dominant in backscattering, as reported by Gao [7]. For thick tissues and confocal geometries where multiple angles are detected simultaneously, these effects of correlations are expected to be smoothed out to some extent. However, in most applications, these spectral changes are not expected to be negligible and spectroscopic measurements of tissues can provide erroneous results. One possible solution for this problem is the simultaneous measurement of morphology (e.g., via QPI) and spectroscopy.

\section{References}

1. E. Wolf, Phys. Rev. Lett. 56, 1370 (1986).

2. M. F. Bocko, D. H. Douglass, and R. S. Knox, Phys. Rev. Lett. 58, 2649 (1987).

3. E. Wolf, Nature 326, 363 (1987).

4. E. Wolf, J. T. Foley, and F. Gori, J. Opt. Soc. Am. A 6, 1142 (1989).

5. A. Dogariu and E. Wolf, Opt. Lett. 23, 1340 (1998).

6. W. R. Gao, J. Biomed. Opt. 15 (2010).

7. W. Gao, Opt. Lett. 35, 862 (2010).

8. M. Hunter, V. Backman, G. Popescu, M. Kalashnikov, C. W. Boone, A. Wax, G. Venkatesh, K. Badizadegan, G. D. Stoner, and M. S. Feld, Phys. Rev. Lett. 97, 138102 (2006).

9. R. Richards-Kortum and E. Sevick-Muraca, Annu. Rev. Phys. Chem. 47, 555 (1996).

10. G. Srinivasan, Vibrational Spectroscopic Imaging for Biomedical Applications (McGraw-Hill, New York, 2010).

11. H. F. Ding, Z. Wang, F. Nguyen, S. A. Boppart, and G. Popescu, Phys. Rev. Lett. 101, 238102 (2008).

12. Z. Wang, L. J. Millet, M. Mir, H. Ding, S. Unarunotai, J. A. Rogers, M. U. Gillette, and G. Popescu, Opt. Express 19, 1016 (2011).

13. Z. Wang, H. Ding, and G. Popescu, Opt. Lett. 36, 1215 (2011).

14. H. Ding, Z. Wang, X. Liang, S. A. Boppart, and G. Popescu, Opt. Lett. 36, 2281 (2011).

15. E. Wolf, Opt. Commun. 1, 153 (1969). 